\title{
CULTURA POLÍTICA DE LOS BOGOTANOS: PATRONES DEL COMPORTAMIENTO POLÍTICO DE CARA A LAS ELECCIONES DE 2019*
}

\section{Political culture of Bogota's citizens: patterns of political behavior ahead of the 2019 elections}

Juan David Cárdenas Ruiz**

Recepción: 17 de febrero de 2020. Aceptación: 12 de junio de 2020

DOI: http://dx.doi.org/10.21017/Rev.Repub.2020.v29.a91

\section{RESUMEN}

El Observatorio de Medios de la Universidad de La Sabana realizó en septiembre de 2019 un estudio de cultura política de los habitantes de Bogotá. Se entrevistó a 781 personas para identificar sus imaginarios frente a la política, sus hábitos de participación, información y socialización política, y sus valores políticos, en medio del contexto electoral regional de octubre de 2019 en la ciudad. Se logró evidenciar un patrón de comportamiento que encierra una contradicción entre un alto nivel de participación electoral histórico y unos bajos niveles de interés por la política, la información sobre temas públicos y la interacción política con otras personas, así como también un imaginario negativo frente a la política y la realidad de la ciudad.

Palabras clave: cultura política, participación política, comunicación política, socialización política, ideología.

* Artículo producto de la investigación «Cultura política de los habitantes de Bogotá de cara a las elecciones de 2019», desarrollado en el marco del Seminario de Investigación en Comunicación Pública de la Facultad de Comunicación de la Universidad de La Sabana.

** Politólogo de la Universidad Nacional de Colombia. Especialista en opinión pública y marketing político de la Universidad Javeriana. Magíster en estudios políticos de la Universidad Nacional de Colombia-Instituto de Estudios Políticos y Relaciones Internacionales (IEPRI). Docente y jefe del Departamento de Comunicación Pública de la Facultad de Comunicación de la Universidad de La Sabana e investigador del Observatorio de Medios adscrito a la misma facultad. Correo electrónico: juancar@unisabana.edu.co 


\begin{abstract}
In September 2019, the Media Observatory of the University of La Sabana conducted a study of political culture of the inhabitants of Bogotá. 781 people were interviewed to identify their imaginary about politics, their habits of participation, information and political socialization, and their political values, in the middle of the regional electoral context of October 2019 in the city. It is possible to demonstrate a pattern of behavior that encloses a contradiction between a high level of historical electoral participation and low levels of interest in politics, information on public issues and political interaction with other people, as well as a negative imaginary regarding politics and the reality of the city.
\end{abstract}

Keywords: political culture, political participation, political communication, political socialization, ideology.

\title{
INTRODUCCIÓN
}

Bogotá, capital de Colombia, es una ciudad que puede ser catalogada como diferente dentro del contexto nacional en términos de su cultura política. Con casi 10 millones de habitantes, conformada por personas de todo el país y de otros países, la ciudad, desde la Constitución Política de 1991, se ha convertido en un escenario político volátil donde los partidos tradicionales fueron perdiendo su peso electoral, al punto que logró tener 3 gobiernos consecutivos de centro izquierda y hoy en día sigue siendo una de las pocas regiones que nunca han podido ser gobernadas por el uribismo como tendencia política predominante de los últimos 16 años en Colombia. Epicentro de la política nacional, las instituciones y los principales hechos de protesta y movilización social, Bogotá se convierte en un fenómeno de permanente análisis desde la cultura política.

Las elecciones del 27 de octubre marcan un nuevo proceso político en donde los bogotanos acudirán a las urnas a elegir a su nuevo alcalde, sus concejales y sus ediles de las juntas administradoras locales. Por encima del promedio nacional de participación, Bogotá es una de las ciudades del país con la más baja tasa de abstención. En el 2016, para la votación del plebiscito, la tasa de participación en Bogotá fue del 46,3\% frente a un 37,4\% nacional. Para las elecciones presidenciales del 2018 la tasa de participación fue del 64,9\% frente a un $53,3 \%$ a nivel nacional, y en la consulta anticorrupción de agosto de 2018 la tasa promedio de participación en la ciudad fue del 45,7\% frente un 32,04\% a nivel nacional (Registraduría General del Estado Civil, 2019).

Detrás de este fenómeno de alta participación electoral surgen inquietudes frente a los elementos de cultura política que activan y subyacen a dicho com- 
portamiento, y que obedecen a procesos de carácter estructural y a fenómenos de naturaleza coyuntural. Teniendo en mente este panorama y queriendo trascender la «encuestocracia» (Huneeus Madge, 2007) en un entorno electoral asfixiado por el exceso de información y el cuestionamiento metodológico a la avalancha de encuestas, el Observatorio de Medios quiso salir a indagar los elementos de la cultura política de los bogotanos de cara a las elecciones del 2019, no con el objetivo de revelar intenciones de voto o posicionamiento de imagen de los candidatos, sino tratando de encontrar patrones comportamentales detrás de las decisiones de una campaña marcada por la volatilidad en las preferencias de los electores, la agresividad y la negatividad de las campañas de los candidatos y el exceso de insumos estadísticos desde las encuestas en los medios y en las redes sociales que han viciado el proceso democrático, alejándolo de su naturaleza deliberativa, racional y política para pasarlo a un plano emocional desprovisto de reflexión y análisis temático.

\section{TRAS LAS HUELLAS DE LA CULTURA POLÍTICA}

El análisis del comportamiento político ha sido un tema de primer orden en la investigación social. Desde el clásico estudio de Verba y Almond, The civic culture: Political attitudes and democracy in five nations (1963), el comportamiento humano frente a los asuntos públicos ha venido siendo estudiado desde distintas perspectivas, buscando establecer patrones de comportamiento y sus determinantes sociales, económicos, políticos, culturales y psicológicos, entre otros. Como lo plantea Heras:

El concepto de cultura política, no obstante su carácter controvertido, ha sido fuente de crecimiento de la ciencia política y se ha nutrido incluso de otras corrientes de investigación principalmente sociológica. Si bien los dos campos teóricos desde los cuales se ha abordado el tema están constituidos por la política comparada y la sociología interpretativa, la literatura más reciente apunta en una dirección mucho más comprensiva, que abarca ya el cambio de valores de la sociedad contemporánea (Heras Gómez, 2002, p. 289).

Esto mismo es corroborado por López de la Roche, quien sostiene que

la noción de cultura política aborda una pluralidad enorme y compleja de fenómenos. El estudio de cada uno de ellos demanda igualmente diversas aproximaciones, diferentes confluencias interdisciplinarias, así como distintos enfoques metodológicos, a las múltiples disciplinas que concurren al estudio de la cultura política o las culturas políticas: historia de la cultura, sociología política, semiología, antropología política, psicología social, ciencia política, lingüística y los estudios de comunicación de masas (López de la Roche, 2000, p. 99). 
Tres corrientes de investigación han predominado en estos estudios y tienen abordajes que no son necesariamente excluyentes: los estudios del comportamiento, el abordaje desde los valores y la corriente de la sociología interpretativa. La primera corriente, basada en el estudio pionero de Verba y Almond, concibe la cultura política dentro de tres dimensiones: conocimiento, sentimiento y evaluaciones, centrando el estudio del fenómeno sobre qué tanto conocen los ciudadanos de sus sistemas y relaciones políticas, cómo se sienten frente a esto y cómo evalúan dichas instituciones y relaciones. En palabras de los autores: «La cultura política de una nación consiste en la particular distribución entre sus miembros de las pautas de orientación hacia los objetos políticos» (Almond \& Verba, 2007, p. 180). Esto implica que deben ser tomadas en cuenta:

«Orientación cognitiva», es decir, conocimientos y creencias acerca del sistema político, de sus papeles y de los incumbentes de dichos papeles en sus aspectos políticos (inputs) y administrativos (outputs); «orientación afectiva», o sentimientos acerca del sistema político, sus funciones, personal y logros; y «orientación evaluativa», los juicios y opiniones sobre objetos políticos que involucran típicamente la combinación de criterios de valor con la información y los sentimientos (Almond \& Verba, 2007, p. 180).

Para Ronald Ingelhart la cultura política responde a una serie de valores que pueden transformarse o no de manera generacional y que van construyendo unos patrones de relacionamiento entre los ciudadanos y los objetos políticos. Ingelhart afirma:

La cultura política implica que los valores, creencias y habilidades de las personas tienen un fuerte impacto sobre la política, particularmente sobre las instituciones democráticas. Estas orientaciones son relativamente centrales y estables, pero pueden cambiar lentamente, especialmente a través de relevos intergeneracionales. La cultura política varía de una sociedad a otra y es transmitida de generación en generación a través de los procesos de socialización. Sin embargo la experiencia de primera mano de cada generación también moldea su panorama, entonces la cultura puede variar de una generación a otra. La cultura cívica es un síndrome coherente de la satisfacción personal, la satisfacción política, la confianza interpersonal y el apoyo hacia el orden existente (Inglehart, 2006, p. 127).

Otra perspectiva, la sociología interpretativa, propone una perspectiva ascendente de construcción de la cultura política a partir de procesos de socialización política. Eckstein (1988) afirmaba que la cultura política responde a una serie de orientaciones que son construidas a través de procesos de socialización. Al respecto dice: 
Las orientaciones no se adquieren de manera automática; deben ser aprendidas. Entonces, un postulado sobre la socialización cultural debe sostener que las orientaciones son aprendidas a través de la agencia de socializadores externos. El repertorio de cogniciones, sentimientos y esquemas de evaluación que procesan nuestras experiencias en acciones debe ser impartido por vehículos culturales (Eckstein, 1988, p. 791).

Este planteamiento va en la misma línea de la idea del bagaje cultural de Clifford Geertz (1973), donde dichas orientaciones serían tramas de significado que responden a procesos históricos de larga data que van moldeando los valores políticos de una sociedad. Heras sostiene:

La idea central es que mediante la recuperación del bagaje de representaciones, símbolos e instituciones de una sociedad, estudiadas por la sociología interpretativa, es posible establecer las bases o raíces de la cultura política; pero que ello no es suficiente para explicar las cotidianas percepciones y actitudes de la sociedad hacia la política. Que para ello se requiere el estudio comparativo y estadístico, como el que se plantea desde la política comparada (Heras Gómez, 2002, p. 276).

Como se puede observar, hay distintos abordajes frente al concepto de cultura política. Para este estudio es fundamental enfocar conceptualmente el abordaje sobre los procesos de decisión política de los electores bogotanos. La toma decisión política se relaciona con tres procesos previos directamente relacionados con hábitos de cultura política, como son el interés por los asuntos públicos, la información sobre asuntos públicos y la socialización política. Silva y Aquino (2015) sostienen que existen distintos factores que influyen sobre la decisión, algunos de ellos sociodemográficos, como la edad y el género; otros socioeconómicos, como el estrato, el nivel educativo y el empleo; otros más de carácter social, como las identidades culturales, la religión y las pertenencias organizacionales, y otros de carácter político, como la fortaleza o la debilidad de los partidos, de los líderes y de las plataformas ideológicas. Esto, sumado al contexto coyuntural de una elección, resumiría los factores de largo y corto plazo a los que hace referencia el modelo de Michigan en la clásica obra The American Voter de Campbell, Converse, Miller y Stokes (1980).

\section{METODOLOGÍA}

El Observatorio de Medios de la Universidad de La Sabana, grupo de investigación reconocido por Colciencias y con registro del Consejo Nacional Electoral como firma encuestadora avalada, realizó un estudio de cultura política 
durante el periodo del 9 al 16 de septiembre en la ciudad de Bogotá. El trabajo de campo se realizó en 19 de las 20 localidades de la ciudad[1], a un total de 781 personas, con un margen de error de 3,5\% y una confiabilidad del $95 \%$; se trata de una muestra representativa de un universo de 6091367 personas, según la proyección del DANE para el año 2019.

Se diseñó un cuestionario de 30 preguntas que se aplicó de manera presencial en cada una de las localidades en razón a su peso proporcional dentro de la población de la ciudad, de la siguiente manera:

Tabla 1.

Distribución de la muestra

\begin{tabular}{|c|c|c|}
\hline Localidad & Encuestas & Porcentaje \\
\hline Suba & 133 & 17 \\
\hline Kennedy & 120 & 15 \\
\hline Engativá & 80 & 10 \\
\hline Ciudad Bolívar & 74 & 9 \\
\hline Bosa & 62 & 8 \\
\hline Usaquén & 47 & 6 \\
\hline Fontibón & 42 & 5 \\
\hline San Cristóbal & 39 & 5 \\
\hline Usme & 34 & 4 \\
\hline Rafael Uribe Uribe & 31 & 4 \\
\hline Barrios Unidos & 27 & 3 \\
\hline Tunjuelito & 18 & 2 \\
\hline Puente Aranda & 17 & 2 \\
\hline Teusaquillo & 13 & 2 \\
\hline Chapinero & 12 & 2 \\
\hline Antonio Nariño & 10 & 1 \\
\hline Santa Fe & 10 & 1 \\
\hline Mártires & 9 & 1 \\
\hline La Candelaria & 3 & 0 \\
\hline
\end{tabular}

1 No se hizo trabajo de campo en la localidad de Sumapaz por su baja representatividad dentro de la totalidad de la población de la ciudad. 
La muestra se distribuyó proporcionalmente también por género y rango de edad para manejar una mayor representatividad según cifras del DANE. La proporción por género fue de $54 \%$ en mujeres y $46 \%$ en hombres. La proporción por edades fue de 15\% (18 a 24 años), 28\% (25 a 36 años), 22\% (37 a 48 años), 19\% (49 a 59 años) y 16\% (60 años o más).

Una vez realizado el trabajo de campo se sistematizó en el software SPSS, donde se hizo el procesamiento estadístico de la información que reflejan los resultados que se presentan a continuación.

\section{RESULTADOS}

El estudio estuvo dividido en cuatro capítulos que buscaban indagar sobre aspectos centrales de la cultura política de los bogotanos: relación de los bogotanos con la ciudad, valores políticos de los bogotanos, hábitos de información política y patrones de socialización política. A continuación se presentan los principales resultados de cada uno de esos apartados.

\section{Relación de los bogotanos con la ciudad}

El primer aspecto que se indagó en el estudio fue el imaginario de los bogotanos frente a la ciudad. En este punto se instó a los entrevistados a definir la ciudad en una palabra, buscando entender el posicionamiento de diversos conceptos que los ciudadanos relacionan con su vida en la ciudad (figura 1).

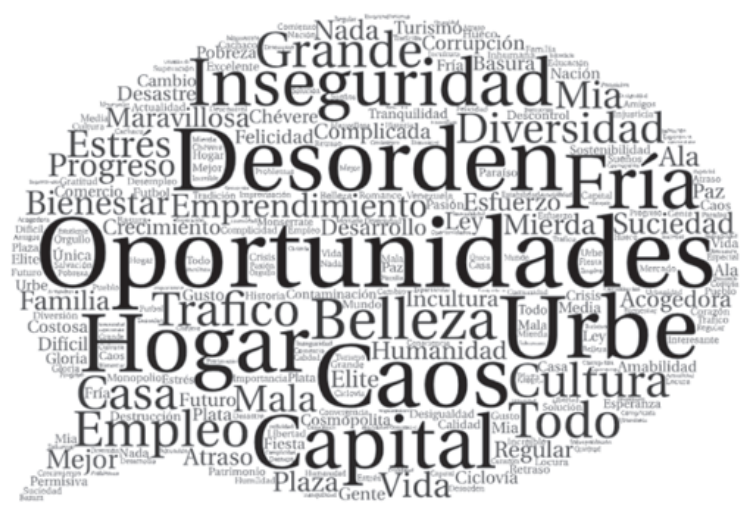

Figura 1. Imaginario de los bogotanos frente a la ciudad

Un gran número de conceptos emergen de este ejercicio. Las palabras más frecuentes muestran una construcción dual del imaginario en una dimensión 
positiva y una dimensión negativa. El 13\% de los encuestados asocian la ciudad con un epicentro de oportunidades, mientras que un $12 \%$ la relaciona con caos y desorden. Un 5\% la considera su hogar, y otro 5\% la cataloga como insegura. Después aparecen con menor peso porcentual adjetivos y referencias a rasgos positivos y negativos de la ciudad. Todos los conceptos se agruparon en 7 categorías con la siguiente distribución:

Tabla 2 .

Imaginario de la ciudad por categorías emergentes

\begin{tabular}{|c|c|c|}
\hline Categoría & Frecuencia & Porcentaje \\
\hline Rasgos culturales y sociales negativos & 243 & 31 \\
\hline Rasgos económicos positivos & 171 & 22 \\
\hline Rasgo culturales y sociales positivos & 160 & 20 \\
\hline Rasgos patrimoniales y turísticos & 109 & 14 \\
\hline Rasgos de identidad y pertenencia & 76 & 10 \\
\hline Rasgos económicos negativos & 16 & 2 \\
\hline Otros conceptos & 6 & 1 \\
\hline Total & 781 & 100 \\
\hline
\end{tabular}

El 52\% de las personas encuestadas afirmó haber vivido en Bogotá durante toda su vida mientras que el $48 \%$ llegó en algún momento de su vida a vivir en la ciudad. Al correlacionar las palabras con el haber vivido o no toda la vida en la ciudad se encuentra significancia estadística al relacionar la ciudad con rasgos económicos positivos en el caso de los que llegaron a vivir a la ciudad y con rasgos de identidad y pertenencia en quienes han vivido toda su vida en la ciudad.

De cara al proceso electoral se formularon dos preguntas relacionadas con el clima de opinión. La primera orientada a establecer la percepción de la actualidad de la ciudad, donde primó el pesimismo con un $44 \%$ de los encuestados opinando que la ciudad iba por mal camino contra un 31\% que consideraba que la ciudad seguía igual y solo un $21 \%$ que consideraba que las cosas iban por buen camino. Estas cifras son consistentes con los niveles de desaprobación del alcalde Enrique Peñalosa que han oscilado entre el 70 y $80 \%$ a lo largo de su mandato. La segunda indagaba sobre las principales problemáticas de la ciudad para el momento de la elección, en donde aparecieron problemas que vienen siendo recurrentes, casi estructurales dentro de la opinión pública capitalina (figura 2). 


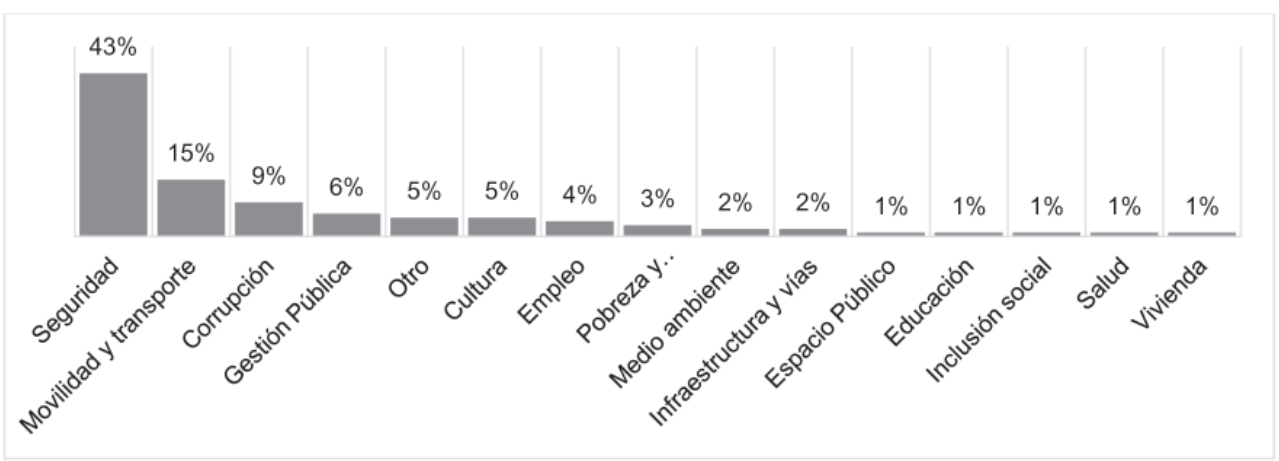

Figura 2. Principal problema para los bogotanos

\section{Los valores políticos de los bogotanos}

El segundo capítulo del estudio se enfocó sobre los valores políticos de los bogotanos. Acá se indagó por el imaginario ciudadano frente a la política, el interés frente a los asuntos públicos, su vínculo con partidos y organizaciones sociales y políticas, autoidentificación e ideología, entre otros aspectos. Un primer hallazgo relevante es el bajo interés que manifiestan tener los encuestados frente a la política. El 34\% de los encuestados no tiene interés alguno y el 18\% solo un poco, seguido de un $23 \%$ que tiene algún interés y solo un 16 y $7 \%$ que manifiestan tener bastante y mucho interés. A esto se le debe agregar el imaginario negativo que se tiene de la política entre los bogotanos (figura 3).

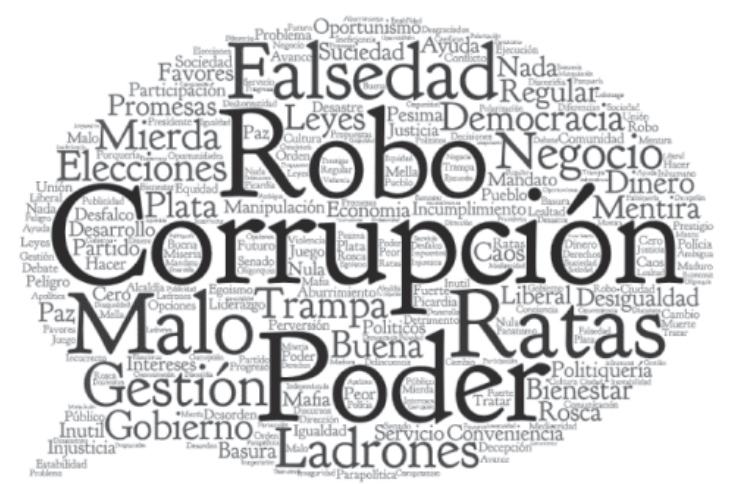

Figura 3. Imaginario de los bogotanos frente a la política

El 48\% asocia la política con la corrupción, seguido de lejos con el 5\% por la palabra robo. La mayoría de las otras palabras son negativas o conceptos genéricos que conciben la política como un asunto netamente institucional. 
Los bogotanos siempre han tenido un promedio de participación electoral superior a la media nacional. En este caso un $57 \%$ afirmó salir siempre a votar, y un $13 \%$ hacerlo casi siempre; es decir, el $70 \%$ tiene un comportamiento electoral activo; sin embargo, a la hora de indagar por la afinidad partidista y la pertenencia organizacional los resultados son críticos. El 74\% manifiesta no sentirse afín a un partido político y un $92 \%$ sostiene no pertenecer a organizaciones sociales, políticas, económicas, religiosas o culturales.

Con respecto al posicionamiento ideológico, los bogotanos en promedio se ubican hacia el centro izquierda con un 4,1 en una escala de 1 a 10, donde 1 corresponde a izquierda y 10 a derecha.

\section{Los hábitos de información y socialización política}

El tercer aspecto que se estudió fueron los hábitos de comunicación política de los bogotanos, buscando indagar sus fuentes de información, sus motivos para consultar dichas fuentes y la frecuencia de esta conducta. Un $51 \%$ afirmó informarse sobre temas políticos mientras el $49 \%$ no lo hace. Dentro de ese $51 \%$ una franja mayoritaria lo sigue haciendo por medios tradicionales (figura 4).

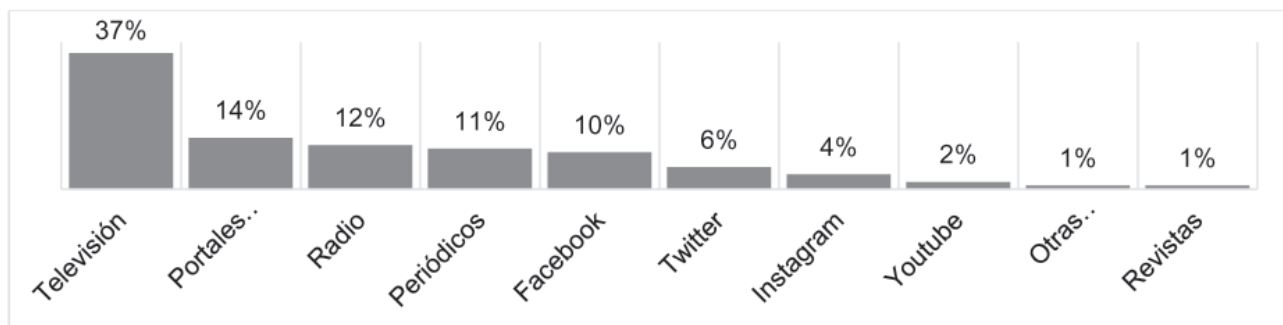

Figura 4. Fuentes de información política

El 37\% lo hace por televisión, el 12\% escucha la radio, 11\% consulta periódicos y $1 \%$ mira revistas. Por otra parte, un $14 \%$ consulta portales de internet, mientras que un $10 \%$ lo hace a través de Facebook, un $6 \%$ por Twitter y un $4 \%$ por Instagram. Esto solo muestra una alternancia en los formatos mas no en las fuentes, ya que los medios consultados a través de redes sociales son los fanpages y perfiles de los medios tradicionales. Por ejemplo, los portales de internet más consultados son los del diario El Tiempo y el diario El Espectador con un $29 \%$ y 19\%, respectivamente. En Facebook la principal fuente, con un $20 \%$, es el perfil de El Tiempo, y sucede lo mismo en las demás redes sociales donde empiezan a aparecer medios nativos digitales pero que aún no logran ser «competencia» para los tradicionales. 
Con respecto a la socialización política, el 51\% de los encuestados manifestó nunca hablar sobre temas políticos con nadie. Por otra parte, el $49 \%$ afirmó hacerlo, siendo los círculos de amigos y la familia, con un $34 \%$ y $25 \%$, respectivamente, los interlocutores más frecuentes de esas conversaciones.

\section{Perfilando al votante bogotano}

Con el objetivo de perfilar el comportamiento electoral de los encuestados, se realizaron una serie correlaciones de variables buscando identificar relaciones de variables que permitan entender dicho comportamiento. La primera correlación estadísticamente significativa identificada se da entre la participación electoral y el interés por la política. Entre menos frecuente es la participación en elecciones, más aumenta el desinterés; aunque se debe mencionar que el desinterés es el comportamiento más común independiente de la frecuencia de la conducta.

Tabla 3.

Correlación entre interés por la política y participación electoral

\begin{tabular}{|c|c|c|c|c|c|}
\hline \multicolumn{2}{|c|}{} & Bajo interés & $\begin{array}{c}\text { Medio } \\
\text { interés }\end{array}$ & Alto interés & Total \\
\hline \multirow{3}{*}{ Usted sale a votar } & Siempre & $42 \%$ & $26 \%$ & $33 \%$ & $100 \%$ \\
& Casi siempre & $55 \%$ & $30 \%$ & $14 \%$ & $100 \%$ \\
& A veces & $74 \%$ & $22 \%$ & $4 \%$ & $100 \%$ \\
& Rara vez & $82 \%$ & $11 \%$ & $6 \%$ & $100 \%$ \\
& Nunca & $75 \%$ & $16 \%$ & $9 \%$ & $100 \%$ \\
\hline
\end{tabular}

N $\quad \mathrm{p}<0,05$

La segunda correlación estadísticamente significativa identificada se da entre la participación electoral y la conducta de información política. Quienes más frecuentemente participan son quienes más se informan sobre política, mientras que quienes menos lo hacen son los que poco o nunca lo hacen.

Tabla 4.

Correlación entre participación electoral e información política

\begin{tabular}{|c|c|c|c|c|}
\hline & & \multicolumn{2}{|c|}{ ¿Se informa sobre temas políticos? } & \multirow{2}{*}{ Total } \\
\hline & & Sí & No & \\
\hline \multirow{5}{*}{ Usted sale a votar } & Siempre & $64 \%$ & $36 \%$ & $100 \%$ \\
\hline & Casi siempre & $50 \%$ & $50 \%$ & $100 \%$ \\
\hline & A veces & $37 \%$ & $63 \%$ & $100 \%$ \\
\hline & Rara vez & $46 \%$ & $54 \%$ & $100 \%$ \\
\hline & Nunca & $29 \%$ & $71 \%$ & $100 \%$ \\
\hline
\end{tabular}

N $\mathrm{p}<0,05$ 
Este patrón se corrobora al comprobar que existe una relación muy fuerte entre el interés por la política y la conducta de información política.

Tabla 5.

Correlación entre interés por la política e información política

\begin{tabular}{|c|c|c|c|c|}
\hline \multicolumn{2}{|c|}{} & \multicolumn{2}{|c|}{ ¿Se informa sobre temas políticos? } & \multirow{2}{*}{ Total } \\
\cline { 3 - 4 } & & Sí & No & \\
\hline \multirow{2}{*}{$\begin{array}{c}\text { Interés por la } \\
\text { política }\end{array}$} & Bajo interés & $34,3 \%$ & $65,7 \%$ & $100,0 \%$ \\
& Medio interés & $62,8 \%$ & $37,2 \%$ & $100,0 \%$ \\
& Alto interés & $88,5 \%$ & $11,5 \%$ & $100,0 \%$ \\
\hline
\end{tabular}

N $\quad$ p $<0,05$

La correlación entre la participación electoral y la socialización política también es significativa. Entre más frecuentemente se participa, con mayor frecuencia se tiende a hablar de política con otras personas.

Tabla 6.

Correlación entre participación electoral y socialización política

\begin{tabular}{|c|c|c|c|c|}
\hline & & \multicolumn{2}{|c|}{$\begin{array}{c}\text { ¿Suele hablar de política con otras } \\
\text { personas? }\end{array}$} & \multirow{2}{*}{ Total } \\
\hline & & Sí & No & \\
\hline \multirow{5}{*}{$\begin{array}{l}\text { Usted sale a } \\
\text { votar }\end{array}$} & Siempre & $58 \%$ & $42 \%$ & $100 \%$ \\
\hline & Casi siempre & $42 \%$ & $58 \%$ & $100 \%$ \\
\hline & A veces & $37 \%$ & $63 \%$ & $100 \%$ \\
\hline & Rara vez & $44 \%$ & $56 \%$ & $100 \%$ \\
\hline & Nunca & $31 \%$ & $69 \%$ & $100 \%$ \\
\hline
\end{tabular}

$\mathrm{N} \quad \mathrm{p}<0,05$

Igualmente se comprueba que el interés por la política suele ser una variable determinante de la socialización política. Para los bogotanos, entre más interés se tiene por la política, más común es sostener conversaciones sobre política con otras personas.

Tabla 7.

Correlación entre interés por la política y socialización política

\begin{tabular}{|c|c|c|c|c|}
\hline \multicolumn{2}{|c|}{} & \multicolumn{2}{|c|}{$\begin{array}{c}\text { iSuele hablar de política con } \\
\text { otras personas? }\end{array}$} & \multirow{2}{*}{ Total } \\
\cline { 3 - 4 } & & Sí & No & $100 \%$ \\
\hline \multirow{2}{*}{$\begin{array}{c}\text { Interés por la } \\
\text { política }\end{array}$} & Bajo interés & $32 \%$ & $68 \%$ & $100 \%$ \\
\cline { 2 - 4 } & Medio interés & $55 \%$ & $45 \%$ & $100 \%$ \\
\cline { 2 - 4 } & Alto interés & $82 \%$ & $18 \%$ & $10 \%$ \\
\hline
\end{tabular}

$\mathrm{N}$ p $<0,05$ 


\section{LA CULTURA POLÍTICA DE LOS BOGOTANOS: REFLEXIONES E INQUIETUDES}

El comportamiento político de los bogotanos, especialmente después de la Constitución de 1991, ha mostrado siempre tener ciertos rasgos de independencia que se han visto materializados en una volatilidad electoral muy alta y en la apertura de espacios de poder local para sectores que a nivel nacional no tienen tanta acogida, especialmente partidos y movimientos de izquierda democrática.

Después de 3 gobiernos de izquierda (Garzón: 2004-2007; Moreno: 2008-2011; Gustavo Petro: 2012-2015) marcados por una transformación política e ideológica, pero a su vez con distintos escándalos y conflictos políticos y judiciales, llegó al poder un candidato de derecha, Enrique Peñalosa, con la promesa de «recuperar Bogotá». En este momento restan 2 meses y medio de gobierno del actual alcalde, cuya popularidad a lo largo del periodo no ha superado el $30 \%$, mientras la ciudad sigue teniendo serios problemas, especialmente en seguridad y movilidad. En el momento de trabajo de campo la ciudad está en plena campaña y los hallazgos deben analizarse en un contexto que combina los elementos estructurales (alcaldías pasadas y la presente) con los coyunturales (campaña y candidatos).

Este estudio busca trascender el análisis basado en encuestas de opinión que arrojan unas tendencias de voto. Se buscó establecer los patrones de comportamiento, las motivaciones y las percepciones ciudadanas que respaldan las decisiones políticas de los bogotanos.

Algo que se debe resaltar es el comportamiento activo frente a los procesos electorales, en donde un 70\% manifestó participar siempre o casi siempre en las elecciones. Sin embargo, el estudio nos permite entrar a analizar el proceso previo a la conducta para problematizar el carácter «racional» de la decisión electoral y dejar planteadas inquietudes para seguir indagando en esa dirección.

A pesar de que un $70 \%$ participa activamente, tan solo un $51 \%$ se informa sobre asuntos públicos, y tan solo un $54 \%$ de ese grupo lo hace todos los días. Igualmente, de ese grupo de participantes activos solo un $49 \%$ habla de política con otras personas; y entre quienes lo hacen, solo el $20 \%$ manifestó hacerlo todos los días. A esto se le debe sumar que en general existe un desinterés ciudadano por los asuntos políticos, de manera que un $52 \%$ de los encuestados están nada o poco interesados por la política. Esto plantea de entrada una contradicción o al menos una pregunta de investigación a desarrollar: ¿Cuáles son los recursos, motivaciones e incentivos que tienen los bogotanos cuando 
en su mayoría no se informan permanentemente, no socializan políticamente de manera frecuente y no tienen un elevado interés en la política y, sin embargo, participan activamente en los procesos electorales?

Otro elemento que se debe tomar en cuenta para tratar de entender la cultura política de los bogotanos reside en los imaginarios que se tienen frente a la política y los medios a través de los cuales, quienes se informan, construyen su opinión frente al acontecer político y sus actores. El hecho de que la mitad de los encuestados asocie inmediatamente la política con el concepto de corrupción y la mayoría de conceptos restantes remitan a aspectos negativos debe sumarse a la ecuación, teniendo en mente el concepto de «espiral del cinismo» (Bennett, 2008; Cappella \& Jamieson, 1996; Erber \& Lau, 1990), o, por el contrario, examinando el concepto de «círculo virtuoso» (Norris, 2001), tratando de indagar el efecto de la información y la socialización política sobre la participación democrática.

En el caso de nuestro estudio, como se evidencia en los resultados, existe una correlación entre los comportamientos positivos (interesarse, informarse y socializar) y una mayor tendencia a participar de las elecciones. Igualmente, un interés más alto en los asuntos públicos se relaciona con una mayor tendencia a informarse y a socializar políticamente. Este hallazgo refuerza la importancia de entender la cultura política desde una perspectiva multidimensional, en donde la educación, la comunicación pública, la pedagogía y la interacción política en distintos espacios son esenciales para el fomento de comportamientos democráticos.

Sin embargo, este comportamiento no se replica en quienes no se informan, no socializan y no se interesan por la política, pero que de todos modos asisten también en un número importante a las urnas.

\section{CONCLUSIONES}

Los procesos electorales son uno de los escenarios donde se pueden ver materializados los cambios en la cultura política de una sociedad.

Para el caso bogotano se evidencian dos fenómenos muy interesantes que deben ser estudiados más a profundidad porque pueden estar marcando el recorrido de la cultura política en construcción que puede ser determinante para el escenario político futuro de la ciudad.

Por una parte se viene dando una consolidación de un electorado cada vez más orientado hacia una agenda de centro izquierda de carácter liberal. Esto, 
sin embargo, no se corresponde con una simpatía partidista específica. La participación electoral parece estar cada vez más mediada por causas o movimientos puntuales, unos de carácter coyuntural y otros de carácter más estructural, que de alguna manera han ido restándole espacio a los partidos tradicionales, por lo menos en lo que a la alcaldía respecta.

Lo curioso es que este tránsito político-ideológico no va acompañado necesariamente de un proceso de sofisticación política, o al menos no por ahora. Siguen siendo muy altos los niveles de desinformación y no muy altos los hábitos de socialización política, por lo menos en los espacios tradicionales. A futuro será interesante volcar la mirada sobre las redes sociales en dos sentidos. Primero, en la medida en que más personas migran hacia ese ecosistema digital y reconfiguran sus hábitos sociales e informativos, será muy importante entender el papel que juega la mediación política en dicho entorno, cada vez más marcado por la polarización, la degradación de la deliberación y las noticias falsas y la desinformación. Segundo, es en este escenario donde se están dando las grandes luchas por el posicionamiento de la agenda pública en el país; y en el marco de lo que seguramente serán los procesos políticos por un amplio periodo de tiempo, en condiciones difíciles de salud pública, estas redes serán el epicentro de la confrontación política en todos los rincones del país.

Finalmente, es destacable cómo los procesos de migración y consolidación de varias generaciones de nuevos bogotanos adoptivos han llevado a una construcción de un imaginario que claramente afecta el ejercicio de las campañas electorales, el discurso político y las estrategias de los actores que participan de lo público en la ciudad.

\section{REFERENCIAS}

Almond, G., \& Verba, S. (2007). La cultura política. En Diez textos básicos de ciencia política (pp. 171-201). Editorial Ariel.

Bennett, W. L. (2008). Changing citizenship in the digital age. In W. L. Bennett (Ed.), The John D. and Catherine T. MacArthur Foundation series on digital media and learning. Civic life online: Learning how digital media can engage youth (p. 1-24). MIT Press.Campbell, A., Converse, P. E., Miller, W. E., \& Stokes, D. E. (1980). The american voter. University of Chicago Press.

Cappella, J. N., \& Jamieson, K. H. (1996). News Frames, Political Cynicism, and Media Cynicism. The Annals of the American Academy of Political and Social Science, $546,71-84$. 
Eckstein, H. (1988). A culturalist theory of political change. American Political Science Review, 82(3), 789-804.

Erber, R., \& Lau, R. R. (1990). Political cynicism revisited: An information-processing reconciliation of policy-based and incumbency-based interpretations of changes in trust in government. American Journal of Political Science, 236-253.

Geertz, C. (1973). The Interpretation of Cultures. New York. Basic Books, Inc,. Publishers.

Heras Gómez, L. (2002). Cultura política: el estado del arte contemporáneo. Reflexión Política, 4(8), 180-191. Retrieved from http://editorial.unab.edu.co/revistas/ reflexion/pdfs/dem_48_4_c.pdf

Huneeus Madge, C. (2007). Democracia vs. Encuestocracia. Mensaje, 56(563), 6-11.

Inglehart, R. (2006). Political culture. In Redefining Culture (pp. 151-160). Routledge.

López de la Roche, F. (2000). Aproximaciones al Concepto de Cultura Política. Convergencia. Revista de Ciencias Sociales, 7(22), 1405-1435.

Norris, P. (2001). A Virtuous Circle? The Impact of Political Communications in PostIndustrial Democracies. Challenges to Democracy, 7247, 100-117. https:/ / doi.org/ $10.1017 /$ S0003055402404339

Registraduría General del Estado Civil. (2019). Sin título. Recuperado en octubre 15, 2019, de https://wsr.registraduria.gov.co/-Historico-de-Resultados,3635-.html

Silva, M. M., \& Aquino, R. S. (2015). Manual de campaña Teoría y Práctica de la Persuasión Electoral. Apuntes Electorales, (9).

Verba, S., \& Almond, G. A. (1963). The civic culture: Political attitudes and democracy in five nations. Princeton Uiversity Press. 